\title{
La producción de la prueba arbitral: las Reglas de la IBA v/s las Reglas de Praga
}

\author{
Juan Eduardo Figueroa Valdés* \\ Juan Eduardo Figueroa Guzmán**
}

Recibido/Received: $31 / 07 / 2020$

Aceptado/Accepted: 29/09/2020

\begin{abstract}
Sumario: 1. Objeto de ambas reglas. 2. Análisis comparativo entre las Reglas de la IBA y las Reglas de Praga. 2.1. En cuanto a la producción de la prueba documental. 2.2. En cuanto a la producción de la prueba testimonial. 2.3. En cuanto a la producción de la prueba pericial. 3. Conclusiones.
\end{abstract}

RESUMEN: En el presente artículo nos referiremos a la prueba en los arbitrajes comerciales internacionales, haciendo un paralelo entre las IBA Rules on the Taking of Evidence in International Arbitration -más conocidas como Reglas de la IBA-y las Rules on the Efficient Conduct of Proceeding in International Arbitrationconocidas coloquialmente como Reglas de Praga-, en relación a la producción de la prueba documental, testimonial y pericial, destacando especialmente los aspectos diferenciadores entre ambas guías.

Palabras Clave: arbitraje internacional, prueba, Reglas IBA, Reglas de Praga.

* Socio de FIHS Abogados. Profesor de post-grado en Facultad de Derecho Universidad de los Andes, Santiago de Chile. Abogado Licenciado en Ciencias Jurídicas de la Universidad de Chile. LL.M. en Derecho Internacional, Comercio, Inversiones y Arbitraje Internacional, Universidad de Heidelberg. Correo electrónico: jef@fihs.cl

** Asociado en FIHS Abogados. Abogado Licenciado en Ciencias Jurídicas de la Universidad de los Andes. Correo electrónico: jfigueroag@fihs.cl

J. E. Figueroa Valdés \& J. E. Figueroa Guzmán, "La producción de la prueba arbitral: las Reglas de la IBA v/s las Reglas de Praga”, Revista Ecuatoriana de Arbitraje, No. 11, 2020, pp. 45-60. 


\title{
Taking evidence in International Arbitration: IBA rules $v / s$ Prague Rules
}

\begin{abstract}
AвSTRACт: In this article we refer to the evidence in international arbitration, doing a parallel between the IBA Rules on the Taking of Evidence in International Arbitration - better known as the IBA Rules- and the Rules on the Efficient Conduct of Proceeding in International Commercial Arbitration colloquially known as the Prague Rules-, in relation to the production of documents, fact witness and expert evidence, especially highlighting the different approach between both guides.
\end{abstract}

KeYwords: international arbitration, evidence, IBA Rules, Prague Rules.

\section{1. Овjeto de ambas Reglas}

Atendido las sofisticadas disputas de común ocurrencia que se producen en la ejecución de los complejos contratos que se celebran hoy en día a nivel global, como también una vez que ya han terminado, sumado a la abundante prueba documental y testimonial que usualmente existe en este tipo de litigios, las Reglas de la IBA y más recientemente las Reglas de Praga ${ }^{1}$, han venido a ser un aporte al tribunal arbitral y a las partes, contribuyendo mediante el otorgamiento de facultades que permiten una administración más eficiente en el manejo de la prueba, en términos de tiempo y $\operatorname{costos}^{2}$.

1. Las Reglas Sobre Tramitación Eficiente en los Procedimientos en el Arbitraje Internacional, más conocidas como Reglas de Praga, fueron concebidas en la IV Conferencia Anual de la Asociación de Arbitraje, que se llevó a cabo en abril de 2017 en Rusia. Tras este evento comenzó el trabajo de 30 representantes de distintas jurisdicciones, especialistas en arbitraje comercial internacional, provenientes mayoritariamente de Europa, especialmente de ex repúblicas soviéticas y de Europa del Este. La versión definitiva fue publicada el 14 de diciembre del año 2018, en el palacio Martinicky, en la ciudad de Praga.

2. D. G. Henríquez, "The Prague Rules: Competitor, Alternative or Addition to the IBA Rules on the Taking of Evidence in International Arbitration?", Asa Bulletin, Swiss Arbitration Association, Vol. 36 No. 2, 2018, p. 351. 
Estas reglas constituyen guías de buenas prácticas, que fijan ciertos estándares internacionales para la producción, presentación y valoración, tanto para la prueba documental, testimonial como pericial, buscando proporcionar un procedimiento eficiente, económico y equitativo para la práctica de la prueba en arbitrajes internacionales ${ }^{3}$. La doctrina ha discutido si deben ser consideradas como guías alternativas o complementarias, aspecto del cual nos ocuparemos al final de este trabajo.

Las Reglas de la IBA, siguiendo la tradición del common law, basado en un principio dispositivo puro ${ }^{4}$, con un enfoque más bien contradictorio en la producción de documentos, testigos y peritos, -adversarial system- atribuyen un rol más pasivo al tribunal arbitral en la administración del arbitraje. Tienen como objetivo disminuir la brecha existente entre los diferentes sistemas legales y sus respectivos procedimientos en la producción de la prueba, en aquellos casos que las partes pertenecen a distintas tradiciones jurídicas ${ }^{5}$, intentado lograr un equilibrio entre el derecho consuetudinario y las tradiciones del derecho civil ${ }^{6}$. El texto es bastante más extenso y detallado que las Reglas de Praga, y su última versión es del año 2010, en la que se hicieron algunos cambios en consideración a los avances tecnológicos, y se incluyeron algunos requisitos en relación a los peritos designados por las partes ${ }^{7}$.

A su turno, las Reglas de Praga parten de la premisa de la adopción de un enfoque del civil law o derecho continental,

3. Reglas de la IBA (International Bar Association) sobre la Práctica de Prueba en el Arbitraje Internacional (2010).

4. H. O. Méndez \& A. M. Médez, Las Reglas de Praga: Recuperación de la tradición jurídica del derecho continental en el procedimiento del arbitraje internacional comercial, p. 31, $<$ https://bit.ly/2HSJOfi> (18/07/2020).

5. Reglas de la IBA (International Bar Association) sobre la Práctica de Prueba en el Arbitraje Internacional, N. 3.

6. D. Jones, Let's get together: Quo Vadis International Construction Arbitration, GAR Live Construction Disputes, conferencia de 9 de julio de 2020.

7. La versión anterior de las Reglas de la IBA sobre Práctica de Prueba en el Arbitraje Internacional data del año 1999. 
dotando al tribunal arbitral de facultades inquisitorias, otorgando un rol proactivo al tribunal en distintos aspectos ${ }^{8}$, a los que nos referiremos posteriormente, tales como, su participación en la etapa de determinación de los hechos ${ }^{9}$, la facultad de proponer una solución transaccional en cualquier etapa del arbitraje $\mathrm{e}^{10}$, o la de determinar las condiciones bajo las cuales se llevará a cabo una audiencia ${ }^{11}$.

Las Reglas de Praga introducen una innovación al facultar al juez para aplicar disposiciones legales que no hayan sido alegadas por las partes, en virtud de la consagración expresa del principio iuria novit curia ${ }^{12}$. Su primera versión fue publicada en diciembre del año 2018, y fue desarrollada por un grupo de abogados provenientes del civil law, con el objeto de mejorar la eficiencia de los procedimientos arbitrales, en vista de que más del 60\% de la población mundial vive en una jurisdicción de derecho civilis.

Su aplicación procede por acuerdo de las partes o por la decisión de oficio del tribunal, una vez oída las partes y prestado su consentimiento o frente al silencio de estas, toda vez que se trata de normas del soft law, en las que prima el principio de autonomía de la voluntad ${ }^{14}$.

8. A modo de ejemplo, en las Reglas de Praga se contempla la obligación del tribunal de celebrar una "reunión preliminar" una vez recibido el expediente, en la que se fijan las reglas del procedimiento que regirá en el arbitraje, dotando al tribunal de facultades para compartir preliminarmente su visión sobre materias del fondo de la disputa, sin que dichos pronunciamientos afecten su imparcialidad e independencia. Por su parte, las Reglas de la IBA regulan una "consulta sobre cuestiones probatorias", instancia en la que se fijan los aspectos probatorios del procedimiento, con un enfoque más procesal, y con menos atribuciones para el tribunal arbitral.

9. Reglas Sobre la Tramitación Eficiente de los Procedimientos en el Arbitraje Internacional (Reglas de Praga) (2018), Artículo 3.

10. Ibídem, Artículo 9.

11. Ibídem, Artículo 8(2).

12. Ibídem, Artículo 7.

13. Universidad de Ottawa, Percentage of the World Population, Civil Law and Common Law systems, $<$ https://bit.ly/3elN1Dv> (10/05/2020).

14. De esa forma deben entenderse el artículo 1(2) de las Reglas de Praga, a pesar de que no lo señala expresamente, limitándose a señalar que el tribunal debe oír a las partes. 


\section{AnÁlisis comparativo entre las Reglas De la IBA y las Reglas de Praga}

A continuación, nos abocaremos a realizar un análisis comparativo entre ambas Reglas, en relación a la producción de la prueba documental, testimonial y pericial, con el objeto de identificar las principales diferencias entres ambas guías o directrices en dichos ámbitos, y poder determinar cuál resulta más adecuada atendido las pretensiones de las partes y la prueba que en cada caso requiere producirse.

\subsection{En cuanto a la producción de la prueba documental}

En las Reglas de la IBA encontramos una disposición bastante amplia relativa a la producción de documentos, al señalar que cada parte presentará todos los documentos que estén a su disposición y sobre los que base sus pretensiones, incluyendo documentos públicos o privados ${ }^{15}$. Como contrapartida a ello, las Reglas de Praga limitan la presentación de documentos, sosteniendo que "[c]omo regla general, se invita al tribunal arbitral y a las partes a evitar cualquier método de exhibición documental, incluido el discovery electrónico"16, en el entendido que esta restricción conlleva una disminución en el número de documentos que se aportan que no necesariamente son relevantes, y que significan mayores costos en términos de eficiencia y tiempo.

En cuanto al requerimiento de exhibición de documentos, creemos que no hay diferencias sustanciales entre las reglas provenientes del common law que establecen que el tribunal arbitral deberá fijar un plazo para que las partes presenten la solicitud de exhibición de documentos, y los procedimientos

15. Reglas de la IBA (International Bar Association) sobre la Práctica de Prueba en el Arbitraje Internacional, N. 3, Artículo 3(1).

16. Reglas Sobre la Tramitación Eficiente de los Procedimientos en el Arbitraje Internaccional (Reglas de Praga), N. 9, Artículo 4(2). 
sujetos a las Reglas de Praga, en los que dicha solicitud se deberá efectuar en la reunión preliminar, y excepcionalmente en una fase posterior, solo si concurren circunstancias que lo ameriten. En ambos sistemas las facultades del tribunal son similares, como también los requerimientos que debe cumplir la solicitud que efectúan las partes, reconociendo los principios de especificidad, relevancia, importancia y proporcionalidad, pero no así las posturas que pueden adoptar frente a esta, toda vez que la Reglas de la IBA contemplan la posibilidad de que aduzcan una objeción a los documentos solicitados por los motivos enumerados taxativamente, dentro de los cuales destacamos, la falta de relevancia suficiente, que suponga una carga excesiva, confidencialidad por razones comerciales o técnicas, entre otros ${ }^{17}$.

Adicionalmente, las Reglas de la IBA contemplan la posibilidad de requerir la exhibición de documentos, en cualquier momento antes de la conclusión del arbitraje, respecto de una persona y organización que no sea parte del mismo $^{18}$, a diferencia de las reglas influenciadas por el derecho continental que exigen que el o los documentos estén en posesión o bajo el control de la parte contraria ${ }^{19}$.

En los últimos años la producción de documentos en instancias arbitrales se ha visto beneficiada por los avances tecnológicos, mediante softwares o herramientas que permiten el almacenamiento digital, una adecuada organización y clasificación de los documentos, y una aportación más ordenada a los procedimientos, que facilitan las tareas de las partes litigantes y de los árbitros, reduciendo las ineficiencias en la producción y aportación de la prueba.

17. Los motivos para que procedan las objeciones son aquellos contemplados en el Artículo 9(2), o la falta de cumplimiento de alguno de los requisitos dispuestos en el Artículo 3(3).

18. Reglas de la IBA (International Bar Association) sobre Práctica de Prueba en el Arbitraje Internacional, N. 3, Artículos 3(9) y 3(10).

19. Reglas Sobre la Tramitación Eficiente de los Procedimientos en el Arbitraje Internaccional (Reglas de Praga), N. 9, Artículo 4(5). 
Es así como, por ejemplo, los actores de los arbitrajes de construcción han sido testigos de esta automatización en la producción documental, reflejado en la implementación de poderosas herramientas, tales como el Modelo BIM (Building Information Modeling), que permite una constitución completa de toda la información del proyecto, siendo un fiel reflejo de la realidad, toda vez que arroja datos objetivos y no sujetos a interpretación; el Critical Path Network (CNP), cuando las disputas involucran elevados montos; el Redfern Schedule, en el cual se registran en una columna los requerimientos de exhibición de documentos, en otra, las objeciones que se planteen por la parte contraria, $y$, en la última columna, las decisiones del tribunal arbitral, ya sea acogiendo $\mathrm{o}$ rechazando la exhibición de los documentos; el Scott Schedule, utilizado en aquellos procedimientos que existen abundantes reclamos, constituyendo un buen resumen de las posiciones de las partes frente a ellos. En la actualidad existen numerosos softwares que han desarrollado algoritmos que facilitan mucho la ubicación de la información contenida en los documentos, que genéricamente ha sido denominada Technology Assisted Review.

Ahora bien, de una revisión de las características de los documentos electrónicos, considerando que en la actualidad la mayoría de los documentos son creados electrónicamente, queda en evidencia que también pueden acarrear complicaciones en la práctica, tales como, un aumento considerable en el volumen del material, que puede implicar un proceso más extenso de búsqueda; la dispersión de los documentos relacionados con un acontecimiento o transacción, en comparación a los documentos en papel, que usualmente se guardan en ciertos lugares físicos limitados; son más vulnerables a ser modificados con propósitos perjudiciales, toda vez que conservar de manera forense una 
cantidad importante de documentos electrónicos conlleva costos significativos ${ }^{20}$.

\subsection{En cuanto a la producción de la prueba testimonial}

De acuerdo a las Reglas de la IBA el tribunal no tiene facultades para limitar el número de testigos que prestarán su testimonio, sin perjuicio de poder requerir la comparecencia de algún testigo ${ }^{21}$; estableciendo que las mismas partes deberán identificar sus testigos ${ }^{22}$. En este aspecto, las Reglas de Praga disponen: "[d]espués de haber oído a las partes, el tribunal arbitral decidirá los testigos que serán citados para prestar declaración en las audiencias [...]" (énfasis añadido) ${ }^{23}$, lo que podría incentivar que las partes presenten exclusivamente los testigos más relevantes para el caso $^{24}$.

Ambos sistemas contemplan la posibilidad de presentar una declaración testimonial escrita, en forma previa a la audiencia probatoria; se diferencian en que las Reglas de Praga sugieren no tener una audiencia, y en la medida que sea posible, resolver la disputa teniendo como base la declaración testimonial, ello con el objetivo de evitar largas audiencias, y dar más eficiencia al proceso, especialmente en aquellos casos en que la declaración es irrelevante, superflua, demasiado gravosa, reiterativa o, innecesaria para la resolución del conflicto $^{25}$, sin perjuicio de que una parte insista en la

20. Sobre el particular, resulta especialmente intertesante el análisis efectuado en el Informe Gestión de la producción de documentos electrónicos de la Cámara de Comercio Internacional (CCI) publicado en agosto de 2016, p. 6 y siguientes.

21. Reglas de la IBA (International Bar Association) sobre Práctica de Prueba en el Arbitraje Internacional, N. 3, Artículo 8(1).

22. Ibídem, Artículo 4(1).

23. Reglas Sobre la Tramitación Eficiente de los Procedimientos en el Arbitraje Internaccional (Reglas de Praga), N. 9, Artículo 5(2).

24. Esta limitación en el número de testigos ha sido parte de las recomendaciones de la CCI para reducir tiempo y costos en el arbitraje, contenidas en ICC Commission Report: Controlling Time and Costs in Arbitration, Second Edition, 2014.

25. Reglas Sobre la Tramitación Eficiente de los Procedimientos en el Arbitraje Internaccional (Reglas de Praga), N. 9, Artículo 5(3). 
comparecencia de un testigo cuya declaración testimonial ya fue aportada, escenario en el cual el tribunal citará al testigo, salvo que concurran razones justificadas para no hacerlo ${ }^{26}$.

Con la misma finalidad de búsqueda de eficiencia y optimización del tiempo, en los arbitrajes que se hubiere acordado aplicar las reglas propias del common law, "el tribunal podrá limitar o excluir cualquier pregunta, respuesta o comparecencia de un testigo, si considerase que dicha pregunta, respuesta o comparecencia es irrelevante, insustancial, irrazonablemente gravosa, repetitiva[...]"27. Además, el tribunal arbitral tiene facultades para no tomar en cuenta una declaración testimonial, en caso de que el testigo no compareciera a declarar en la audiencia respectiva, a menos que, en circunstancias excepcionales, decida lo contrario ${ }^{28}$.

En nuestra opinión, la audiencia probatoria de contra interrogatorios de testigos juega un rol fundamental, y no estimamos conveniente prescindir de ella, toda vez que estos contribuyen a un mejor esclarecimiento de los hechos materia del arbitraje, constituyendo la oportunidad procesal adecuada para obtener una mayor o menor convicción del relato expuesto en la declaración escrita. Es más, creemos que la realización de la audiencia probatoria a través de herramientas tecnológicas, modalidad que es admitida en ambas guías materia de este estudio, con el objeto de reducir los costos asociados a su celebración, tales como gastos de transporte de los abogados, testigos y/o peritos, puede influir negativamente en la convicción del tribunal arbitral, ya que lamentablemente hay una percepción más íntegra de las declaraciones de los participantes cuando esta instancia procesal se desarrolla presencialmente, gracias a la apreciación de la comunicación e

26. Ibídem, Artículo 5(7).

27. Reglas de la IBA (International Bar Association) sobre Práctica de Prueba en el Arbitraje Internacional, N. 3, Artículo 8(2).

28. Ibídem, Artículo 4(7). 
interacción gestual ${ }^{29}$. Con todo, debemos admitir que, a partir de la reciente experiencia de la pandemia del COVID-19, se ha ido y se irá perfeccionando la forma de rendir pruebas testimoniales en forma virtual ${ }^{30}$.

Ambas guías otorgan al tribunal arbitral amplias facultades de control y dirección en la audiencia probatoria ${ }^{31,32}$, pudiendo rechazar la formulación de preguntas, alterar el orden de comparecencia de los testigos y organizar el careo de testigos ${ }^{33,34}$, como también realizar la audiencia mediante videoconferencias o plataformas electrónicas ${ }^{35,36}$. Por su parte, las Reglas de Praga "van más allá y los árbitros podrían tener la confianza de interrumpir los interrogatorios con mucha discrecionalidad" ${ }^{37}$.

La guía de la IBA contempla la posibilidad de que el tribunal arbitral solicite a cualquier persona pruebas orales o escritas sobre cualquier asunto que considere relevante, permitiendo a las partes interrogar al testigo convocado por el tribunal ${ }^{38}$.

$\mathrm{Al}$ respecto, resulta bastante interesante el contenido del artículo 4(3) de la guía clásica del sistema anglosajón, que

29. En el mismo sentido, M. RegGiardo \& E. Dibos, "Las Reglas de Praga no son tan malas como parecen", Forseti Revista de Derecho, Vol. 10, 2019, pp. 7-8.

30. Sobre el particular, resulta de especial relevancia la guía publicada el 9 de abril de 2020 por la CCI sobre medidas para mitigar los efectos de la pandemia: ICC Guidance Note on Possible Measures Aimed at Mitigating the Effects of the Covid-19 Pandemic, $<\mathrm{https} / / / \mathrm{bit} .1 \mathrm{y} / 3 \mathrm{kWmtwj}>$ $(10 / 05 / 2020)$.

31. Reglas Sobre la Tramitación Eficiente de los Procedimientos en el Arbitraje Internaccional (Reglas de Praga), N. 9, Artículos 5(7).

32. Reglas de la IBA (International Bar Association) sobre Práctica de Prueba en el Arbitraje Internacional, N. 3, Artículo 8(2).

33. Reglas Sobre la Tramitación Eficiente de los Procedimientos en el Arbitraje Internaccional (Reglas de Praga), N. 9, Artículo 5(9).

34. Reglas de la IBA (International Bar Association) sobre Práctica de Prueba en el Arbitraje Internacional, N. 3, Artículo 8(2).

35. Ibídem, Artículo 8(2).

36. Reglas Sobre la Tramitación Eficiente de los Procedimientos en el Arbitraje Internaccional (Reglas de Praga), N. 9, Artículo 8(2).

37. M. Reggiardo \& E. Dibos, N. 29.

38. Reglas de la IBA (International Bar Association) sobre Práctica de Prueba en el Arbitraje Internacional, N. 3, Artículo 8(5). 
reza: "[n]o será considerado impropio que una Parte, sus directivos, empleados, asesores legales u otros representantes, entrevisten a sus testigos o potenciales testigos y discutan con ellos sus posibles testimonios" ${ }^{\prime 39}$. Es menester señalar que esta preparación de los testigos no es aceptable usualmente en los regímenes del civil law, situación que se ve reflejada en la no incorporación de una norma análoga en las Reglas de Praga.

\subsection{En cuanto a la producción de la prueba pericial}

De la revisión de las disposiciones contenidas en ambas directrices en relación a los testigos expertos, no nos sorprende que, al igual que en la regulación de las demás materias, las Reglas de la IBA contienen disposiciones más detallistas que las Reglas de Praga. A modo de ejemplo, las primeras regulan pormenorizadamente el contenido del dictamen pericial ${ }^{40}$.

En las Reglas de la IBA los peritos son considerados testigos expertos, lo que implica que expresan sus opiniones en base a su propia experiencia, con el objeto de ayudar al tribunal arbitral en aquellas materias referidas en su informe ${ }^{41}$, a diferencia de un testigo ordinario que declarará sobre los hechos, pero no desde una perspectiva objetiva ${ }^{42}$.

En las dos guías se admite la posibilidad de designar peritos de oficio o por las partes ${ }^{43}$. En la práctica del arbitraje internacional, solo en casos excepcionales es el tribunal el

39. Reglas de la IBA (International Bar Association) sobre Práctica de Prueba en el Arbitraje Internacional, N. 3, Artículo 4(3).

40. Ibídem, Artículos 5(2) y 6(4).

41. De esa misma forma lo entiende el Chartered Institute of Arbitrators Protocol for the Use of Party-Appointed Expert Witnesses in International Arbitration, Artículos 4(3) y 8(a), <https:// bit.ly/32LoiF6> (10/05/2020).

42. Tratándose de los peritos designados por las partes, el profesor D. JonEs, N. 6, p. 11, reconoce que existen importantes desafíos en cuanto a la objetividad con la que deben actuar, toda vez que su labor es ayudar al tribunal y no a la parte que los nombró. En el mismo sentido se puede consultar M. KANTOR, "A Code of Conduct for Party-Appointed Experts in International Arbitration", Arbitration International, Vol. 26, No. 3, p. 323 y siguientes.

43. En nuestra opinión las partes pueden ahorrar bastante tiempo y dinero si el tribunal arbitral designa el perito, además de que muchas veces se puede focalizar en forma más útil la prueba. 
que designa los peritos, ya que usualmente solo se producen peritajes de parte ${ }^{44}$. En la guía proveniente del common law, se establece que, en el escenario que un perito designado por la parte, cuya comparecencia a la audiencia probatoria haya sido solicitada, no lo hiciera, el tribunal arbitral podrá descartar el dictamen pericial, a menos que en circunstancias excepcionales determine lo contrario ${ }^{45}$; mientras que en las reglas influenciadas por el principio inquisitorio, la actuación del perito se puede limitar a la entrega de su informe escrito, sin perjuicio de la posibilidad de que sea convocado para ratificar su testimonio en la audiencia respectiva ${ }^{46}$.

Ahondando en los testigos expertos nombrados por las partes, cabe hacer mención que es crucial la declaración de independencia de estos, cuestión que se encuentra recogida en las Reglas de la IBA ${ }^{47}$, como también en el CIArb Protocol ${ }^{48}$, que en su artículo 8 indica los requisitos que debe cumplir el testigo experto para declarar que su evidencia no ha sido influenciada por las presiones del proceso ni por alguna de las partes.

Al tratarse del dictamen pericial, cuando el testigo experto es designado por el tribunal, en los procedimientos sujetos a las reglas propias del sistema adversarial, el perito, antes de aceptar su nombramiento, también deberá pronunciarse

44. T. Chelmick \& G. Spalton, "Organisation of the Proceedings in Construction Arbitrations", en S. Brekoulakis \& D. Brynmor Thomas (Eds.), The Guide to Construction Arbitration, Global Arbitration Review, Second Edition, 2018, p. 164.

45. Reglas de la IBA (International Bar Association) sobre Práctica de Prueba en el Arbitraje Internacional, N. 3, Artículo 5(5).

46. Reglas Sobre la Tramitación Eficiente de los Procedimientos en el Arbitraje Internaccional (Reglas de Praga), N. 9, Artículo 6(4).

47. De acuerdo al artículo 5(2) de las Reglas de la IBA el dictamen del perito designado por una parte deberá contener una declaración acerca de su independencia respecto a las partes, sus asesores legales y el tribunal arbitral, como también una descripción de las instrucciones que ha recibido para evacuar sus opiniones y conclusiones, disposiciones que fueron incorporadas en la versión más reciente de la guía.

48. Chartered Institute of Arbitrators, Protocol for the Use of Party-Appointed Expert Witnesses in International Arbitration, N. 41. De acuerdo a lo expuesto en su prefacio, amplía las Reglas de la IBA, toda vez que brinda una guía más detallada sobre lo que debería estar en la opinión escrita de un experto, entre otros aspectos de los que se ocupa más minuciosamente. 
sobre su cualificación e independencia ${ }^{49}$, y las partes, a su turno, deberán informar si tienen alguna objeción sobre dicho pronunciamiento. El informe del perito será enviado a las partes, las que podrán examinarlo y contestarlo mediante la presentación de un escrito, de una declaración testimonial o mediante otro dictamen pericial de un perito designado por la parte $^{50}$. Resulta especialmente interesante las facultades que se otorgan al testigo experto en estas circunstancias, toda vez que estará dotado de la misma autoridad que el tribunal arbitral para solicitar acceso a la información ${ }^{51}$.

A propósito del nombramiento del perito designado por el tribunal arbitral, cabe destacar el deber de recabar las sugerencias de las partes sobre el nombramiento, a pesar de que dichas recomendaciones no tengan carácter vinculante para el tribunal ${ }^{52}$.

Ahora bien, ambas reglas contemplan la posibilidad de efectuar una reunión de peritos, por instrucción del tribunal, -en caso de las Reglas de la IBA procede solo tratándose de peritos designados por las partes, a diferencia de las Reglas de Praga que no establecen esa limitación-, con el objeto de que se pronuncien sobre aquellos puntos cuyas posturas coinciden, como también aquellos en que discrepen, y en este caso, las razones del desacuerdo ${ }^{53,54,55}$.

49. Reglas de la IBA (International Bar Association) sobre Práctica de Prueba en el Arbitraje Internacional, N. 3, Artículo 6(2).

50. Ibídem, Artículo 6(5).

51. Ibídem, Artículo 6(3).

52. Reglas Sobre la Tramitación Eficiente de los Procedimientos en el Arbitraje Internaccional (Reglas de Praga), N. 9, Artículo 6(2)(a).

53. Reglas de la IBA (International Bar Association) sobre Práctica de Prueba en el Arbitraje Internacional, N. 3, Artículo 5(4).

54. Reglas Sobre la Tramitación Eficiente de los Procedimientos en el Arbitraje Internaccional (Reglas de Praga), N. 9, Artículo 6(7).

55. La reunión de peritos es una práctica recomendada por la CCI para reducir tiempo y costos en el arbitraje, de acuerdo al ICC Commission Report: Controlling Time and Costs in Arbitration, 2014, <https://bit.ly/3elRcjV> (11/05/2020). 
Asimismo, en el contexto de las Reglas de la IBA, durante la audiencia probatoria el tribunal arbitral, las partes, y los peritos designados por estas últimas, podrán interrogar al testigo experto designado por el tribunal sobre cuestiones surgidas en su dictamen, en las presentaciones de las partes o en los informes de los peritos de parte ${ }^{56}$. En lo concerniente a este aspecto, desafortunadamente no existe norma análoga en las Reglas de Praga, atendido a las restricciones para la realización de la audiencia probatoria, sin perjuicio de que el perito pueda ser convocado a ratificar sus conclusiones, de conformidad a lo expuesto precedentemente, $\mathrm{y}$ a la posibilidad de que se acuerde una lista de preguntas sobre el contenido de su informe, que contemple las cuestiones cuya revisión se considere necesaria ${ }^{57}$.

Otra práctica frecuente, y en nuestra opinión de bastante utilidad, tratándose de informes de testigos expertos contradictorios, es el empleo del mecanismo de hot tubbing, consistente en que el tribunal arbitral escucha a los distintos expertos en forma simultánea acerca de temas específicos, de modo que cada experto pueda responder a los puntos levantados por otros expertos, lo que facilita la comprensión de las diferencias por parte del tribunal arbitral en forma más rápida y eficiente.

Frente a informes periciales contradictorios, creemos que el tribunal arbitral, en su labor de búsqueda de la verdad, debe analizar la armonía que guarden con las demás pruebas rendidas en el proceso, los antecedentes que considero cada testigo experto, y cuales son las buenas prácticas de la industria.

Por último, y sin intenciones de acabar esta interesante temática, estimamos bastante útiles las buenas prácticas

56. Reglas de la IBA (International Bar Association) sobre Práctica de Prueba en el Arbitraje Internacional, N. 3, Artículo 8(3)(d).

57. Reglas Sobre la Tramitación Eficiente de los Procedimientos en el Arbitraje Internaccional (Reglas de Praga), N. 9, Artículo 1(2). 
sugeridas por el profesor JONES en su reciente publicación ${ }^{58}$, aplicables en aquellos casos que participa más de un testigo experto, que al igual que las reglas analizadas en el presente artículo, apuntan al aumento de eficiencia y la reducción de los costos, a través de la reducción e identificación de las diferencias en los distintos dictámenes periciales; a saber ${ }^{59}$ :

(i) Identificar las disciplinas que requieren evidencia experta, y los testigos expertos propuestos.

(ii) Establecer dentro de cada disciplina una lista común de preguntas.

(iii) Diferir la producción de los informes de los testigos expertos hasta que toda la evidencia objetiva, esto es, prueba documental y testimonial, esté disponible.

(iv) Exigir a los testigos expertos de cada disciplina que produzcan un informe conjunto, identificando las áreas de acuerdo y de desacuerdo.

(v) Exigir a los testigos expertos de cada disciplina que produzcan un informe individual solo en aquellas áreas que hay desacuerdo.

(vi) Exigir a los testigos expertos que produzcan informes de "respuesta" sobre la base de los montos no disputados.

\section{Conclusiones}

A modo de conclusión estimamos menester señalar que la aplicación de una u otra regla dependerá de cada caso en particular, de las tradiciones jurídicas de las partes

58. D. Jones, N. 6, p. 13.

59. Traducción libre. Texto original: "1.- First, identify disciplines in need of expert evidence and experts proposed; 2.- Second, establish within each discipline a common list of questions; 3.Third, defer the production of all expert reports until all factual evidence (documentary and witness) is available; 4.- Fourth, require the experts within each discipline to produce a joint expert reports identifying areas of agreement and disagreement; 5.- Fifth, require the experts within each discipline to produce individual experts reports on areas on disagreement only; and 6.- Sixth, require the experts to produce "reply" experts reports conducted on a "figures-as-figures" basis". 
involucradas, como también del rol más o menos activo que se quiera otorgar al tribunal arbitral.

El primer borrador de las Reglas de Praga, erróneamente a nuestro parecer, indicaba que nacían como respuesta a las Reglas de la IBA y sus problemas, por lo que se entendía que venían a ser una suerte de reemplazo u opción. Afortunadamente, en la versión definitiva, se eliminó esa alusión, y se agregó la siguiente mención: "[1]a finalidad de las Reglas de Praga no es reemplazar las ya facilitadas por varias instituciones, sino que están diseñadas para complementar el procedimiento acordado por las partes o para ser aplicadas por los tribunales arbitrales en una controversia específica" ${ }^{60}$. De igual forma, el prólogo de las Reglas de la IBA sostiene: "[1]as Reglas están diseñadas para ser usadas y adoptadas conjuntamente con reglas institucionales, ad-hoc $u$ otras reglas o procedimientos que puedan resultar de aplicación en arbitrajes internacionales".

De acuerdo a nuestro parecer, al tratarse de normas de soft law, y considerando que son guías o directrices, lo óptimo es adecuarlas para cada caso, atendido a las particularidades que pueden tener, tomando ciertos aspectos de la guía que sigue la tradición del common law y otros de aquella familiarizada con el civil law, de modo tal que la producción de la prueba se ajuste a las circunstancias propias de cada disputa.

Por último, no hay que dejar de mencionar, que las Reglas de la IBA han tenido una amplia aceptación en la comunidad internacional, lo que queda en evidencia en su adopción en numerosos procedimientos, en las casi cuatro décadas que han transcurrido desde la publicación de su primera versión ${ }^{61}$. Las Reglas de Praga, por su parte, están recién zarpando su viaje, por lo que el tiempo dirá la acogida que tendrán.

60. Reglas Sobre la Tramitación Eficiente de los Procedimientos en el Arbitraje Internaccional (Reglas de Praga), N. 9.

61. Las Reglas Suplementarias de la IBA que Regulan la Presentación y Recepción de Prueba en el Arbitraje Comercial Internacional fueron originalmente dictadas en 1983. 